\title{
SINTOMAS URINÁRIOS IRRITATIVOS APÓS PARTO VAGINAL OU CESÁREA
}

\author{
Kátia Pary Scarpa ${ }^{1}$, Viviane Herrmann ${ }^{2 *}$, Paulo Cesar Rodrigues Palma ${ }^{3}$, Cassio Luis Zanettini Riccetto ${ }^{4}$, Sirlei Siani Morais ${ }^{5}$
}

Trabalho realizado na Universidade Estadual de Campinas - UNICAMP, Campinas, SP

\author{
*Correspondência: \\ Rua Alexandre Fleming 101 - \\ Cidade Universitária Zeferino \\ Vaz - Caixa Postal 6081 \\ Campinas - SP \\ CEP 13083-970
}

\begin{abstract}
RESUMO
OвJEтIvo. Avaliar a frequência de Sintomas Urinários Irritativos (SUI) três anos após o parto em mulheres previamente entrevistadas no terceiro trimestre da gestação e sua associação com a via de parto exclusiva, a paridade, a idade materna, o peso do RN, a realização da episiotomia e o uso de fórcipe. MÉTodos. Das 340 mulheres, originalmente avaliadas no terceiro trimestre da gestação, 120 foram localizadas e entrevistadas por telefone, três anos após o parto, entre junho e outubro de 2006 . Foi analisada a associação entre SUI e via exclusiva de parto (vaginal ou cesáreo), paridade, idade materna, peso do recém-nascido, realização da episiotomia e uso de fórcipe. Os resultados foram avaliados por meio de estatística descritiva, teste Qui quadrado e cálculo da razão de prevalência $(p<0,05)$. Resultados. 0 grupo de mulheres estudadas consistiu de primíparas (37,5\%) e multíparas $(62,5 \%)$. As 95 que tiveram via exclusiva de parto foram categorizadas em vaginal (53) e cesáreas (42). Não houve associação significativa entre a presença de SUI após o parto e a via de parto exclusiva e a paridade. Também não encontramos associação entre a presença de SUI e a idade materna ( $>35$ ) peso do recém-nascido ( $\geq 4000 \mathrm{~g}$ ), realização da episiotomia e uso de fórcipe.

Conclusã̃o. A via de parto e a paridade não foram fatores determinantes para a disfunção do trato urinário inferior após o parto, representada pelos sintomas urinários irritativos, tampouco, a idade materna, o peso do RN, a realização da episiotomia e o uso de fórcipe.
\end{abstract}

Unitermos: Sintomas Urinários Irritativos. Paridade. Parto obstétrico. Cesárea. Episiotomia.

\section{INTRODUÇÃO}

Há evidências na literatura de que o trauma obstétrico pode causar vários graus de lesões nas estruturas neuromusculares do assoalho pélvico ${ }^{1}$. A lesão isquêmica do nervo pudendo, com subsequente denervação da musculatura estriada periuretral, diminuindo a pressão intra-uretral, pode determinar a ocorrência de Sintomas Urinários Irritativos (SUI) após o parto².

O efeito protetor da episiotomia sobre o assoalho pélvico, segundo a literatura, é controverso. Outros fatores como o uso do fórcipe ${ }^{4}$ e o peso maior do recém-nascido (RN) ( $\geq 4000 \mathrm{~g}$ ) parecem acentuar a tensão no assoalho pélvico, durante 0 período expulsivo, promovendo lesões nas suas estruturas, inclusive no esfíncter anal ${ }^{5,6}$. Quanto à idade materna ( $\geq 35$ anos), também foi identificada como fator determinante para a incidência de sintomas do trato urinário inferior após o parto ${ }^{6}$.

Além da via de parto vaginal, alterações anatômicas e fisiológicas que ocorrem no trato urinário inferior ao longo da gestação predispõem o desencadeamento de SUI. Os SUI, decorrentes da gestação ou do trauma obstétrico, podem persistir após o parto 7,8 e gestações subseqüentes parecem atribuir risco adicional à disfunção do trato urinário inferior, aumentando a severidade dos sintomas urinários ${ }^{9}$. Scarpa et al. ${ }^{10}$ observaram que a paridade (multíparas $\geq 4$ ) foi associada ao aumento de SUI, particularmente da noctúria e freqüência miccional, durante o último trimestre da gestação.

A cesariana eletiva tem sido associada ao menor risco de incidência de sintomas urinários, quando comparada ao parto vaginal ${ }^{11}$. Entretanto, o efeito protetor da cesariana eletiva, a longo prazo, permanece controverso ${ }^{12}$.

O Brasil, entre os países do mundo, apresentou índices elevados de cesarianas na década de 1980, alcançando alta prevalência particularmente em nulíparas ${ }^{13}$. Segundo o Ministério da Saúde do Brasil, o índice de cesariana no Sistema Único de Saúde diminuiu de 32,4\% em 1995 para 26,4\% em $2003^{14}$. Entretanto, na saúde particular, a taxa de parto cesárea é de $70 \%$, sendo, muitas eletivas sem justificativa precisa ${ }^{15}$. Acredita-se que a prevenção de distúrbios urinários decorrentes do parto vaginal tem sido uma justificativa para o aumento da prática de cesarianas no mundo todo ${ }^{16}$.

1. Pós-graduanda do departamento de Tocoginecologia da Faculdade de Ciências Médicas - FCM da Universidade Estadual de Campinas - Unicamp, Campinas, SP

2. Professora associada do departamento de Tocoginecologia da Faculdade de Ciências Médicas - FCM da Universidade Estadual de Campinas - Unicamp, Campinas, SP

3. Professor titular do departamento de Cirurgia da Faculdade de Ciências Médicas - FCM da Universidade Estadual de Campinas - Unicamp, Campinas, SP

4. Professor associado do Departamento de Cirurgia da Faculdade de Ciências Médicas - FCM da Universidade Estadual de Campinas - Unicamp, Campinas, SP

5. Estatística do departamento de Tocoginecologia da Faculdade de Ciências Médicas da Universidade Estadual de Campinas - Unicamp, Campinas, SP 
O objetivo deste estudo foi avaliar a prevalência de sintomas urinários irritativos após o parto e sua associação com a via exclusiva de parto, paridade, idade materna, peso do $\mathrm{RN}$, realização da episiotomia e uso de fórcipe em mulheres da região de Campinas/SP.

\section{MÉTOdos}

Este estudo foi analítico de corte transversal. Inicialmente, 340 mulheres, na $26^{\mathrm{a}}$ semana de gravidez ou mais, foram selecionadas no Ambulatório de Pré-Natal do Hospital de Clínicas da Universidade Estadual de Campinas (Unicamp), entre junho e outubro de 2003. As gestantes foram convidadas pela fisioterapeuta a participar da entrevista, respondendo individualmente ao questionário pré-testado, contendo perguntas sobre SUI no último trimestre da gestação. Segundo o Termo de Consentimento Livre e Esclarecido, autorizaram contato telefônico futuro. Três anos após o parto, entre junho e outubro de 2006, das 340 mulheres originalmente entrevistadas, 133 foram encontradas por telefone e destas, 120 foram entrevistadas pela pesquisadora principal. O segundo questionário aplicado tinha perguntas prétestadas sobre SUI após o parto e dados obstétricos. As mulheres aceitaram participar do estudo voluntariamente.

Apesar de contatadas, 13 foram excluídas do estudo por: duas tiveram parto gemelar, cinco encontravam-se grávidas no momento da entrevista atual e seis tiveram outro parto entre a primeira entrevista e a atual. Outros critérios de exclusão foram a presença de: diabetes, infecção do trato urinário inferior, cálculo renal e uso de medicamentos que interferem na função do trato urinário inferior no momento da entrevista atual e tratamento prévio para os sintomas urinários, cirúrgico ou conservador (realização de exercícios da musculatura do assoalho pélvico), entre a primeira entrevista e a atual.

Este estudo foi aprovado pelo Comitê de Ética em Pesquisa da Faculdade de Ciências Médicas da Unicamp (protocolo No247/ 2006)

Os sintomas urinários irritativos: frequência miccional, noctúria, urgência, urge-incontinência e enurese noturna foram considerados indicadores subjetivos de alterações percebidas pela mulher e definidos segundo a Sociedade Internacional de Continência ${ }^{17}$.

Para análise foram considerados: via exclusiva de partos anteriores, vaginal ou cesárea e paridade, categorizada em primíparas, multíparas com $2-3$, multíparas $\geq 4$. Outras variáveis avaliadas foram a idade materna, em anos, na data do parto $(<25,25-35,>35)$, o peso do RN (< 4000g; $\geq 4000 \mathrm{~g})$, a realização da episiotomia e o uso de fórcipe. Os resultados foram avaliados por meio de estatísticas descritivas e testes Qui quadrado e cálculo de razão de prevalência (bruta e ajustada). O software utilizado para análise foi o SAS versão 8.2 e o nível de significância assumido foi de $5 \%{ }^{18}$.

\section{Resultados}

Segundo as características das 120 mulheres entrevistadas três anos após o último parto, 40\% não completaram o ensino fundamental, mas $34,2 \%$ e $5,8 \%$ tinham o ensino médio e superior completos, respectivamente. A maioria $(79,1 \%)$ vivia em união consensual ou era casada. Quanto à etnia, $47,5 \%$ se

\begin{tabular}{|c|c|c|c|}
\hline \multicolumn{4}{|c|}{$\begin{array}{c}\text { Tabela } 1 \text { - Distribuição percentual dos sintomas urinários } \\
\text { irritativos (SUI) após o parto segundo a via exclusiva } \\
\text { de parto, vaginal ou cesárea }(n=95)\end{array}$} \\
\hline SUI após o parto & $\begin{array}{l}\begin{array}{l}\text { Vaginal } \\
(\mathrm{n}=53) \\
\mathrm{n}(\%)\end{array}\end{array}$ & $\begin{array}{l}\text { Cesárea } \\
\frac{(\mathrm{n}=42)}{n(\%)}\end{array}$ & Valor-p \\
\hline $\begin{array}{l}\text { Urgência } \\
\text { Sim } \\
\text { Não }\end{array}$ & $\begin{array}{l}25(47,2) \\
28(52,8)\end{array}$ & $\begin{array}{l}15(35,7) \\
27(64,3)\end{array}$ & 0,0894 \\
\hline $\begin{array}{l}\text { Urge-incontinência } \\
\text { Sim } \\
\text { Não }\end{array}$ & $\begin{array}{l}21(39,6) \\
32(60,4)\end{array}$ & $\begin{array}{l}15(35,7) \\
27(64,3)\end{array}$ & 0,6999 \\
\hline $\begin{array}{l}\text { Frequência miccional } \\
\text { Sim } \\
\text { Não }\end{array}$ & $\begin{array}{l}13(24,5) \\
40(75,5)\end{array}$ & $\begin{array}{l}10(23,8) \\
32(76,2)\end{array}$ & 0,9353 \\
\hline $\begin{array}{l}\text { Noctúria } \\
\text { Sim } \\
\text { Não }\end{array}$ & $\begin{array}{l}26(49,1) \\
27(50,9)\end{array}$ & $\begin{array}{l}17(40,5) \\
25(59,5)\end{array}$ & 0,4040 \\
\hline $\begin{array}{l}\text { Enurese noturna } \\
\text { Sim } \\
\text { Não }\end{array}$ & $\begin{array}{c}4(7,5) \\
49(92,5)\end{array}$ & $\begin{array}{c}5(11,9) \\
37(88,1)\end{array}$ & 0,2120 * \\
\hline
\end{tabular}

Teste Qui quadrado

*Teste Exato de Fisher

autodeclararam não brancas; destas, a maioria se considerava parda e somente $6,7 \%$ negras. A idade das mulheres variou entre 19 e 43 anos, sendo que 45\% tinham menos de 25, 43,3\% entre 25 e 35 e somente $11,7 \%$ acima de 35 anos. Quanto à paridade, $37,5 \%$ eram primíparas e $62,5 \%$ multíparas, destas $50 \%$ tinham até três partos anteriores e $12,5 \%$ quatro ou mais.

As mulheres que tiveram via única de partos anteriores foram categorizadas em via de parto exclusivamente vaginal (53) e exclusivamente cesárea (42). Três anos após o último parto, em mulheres que tiveram via exclusiva de parto vaginal, a prevalência da noctúria, urgência e urge-incontinência foi de 49,1\%, $47,2 \%$ e 39,6\%, respectivamente. Em mulheres submetidas exclusivamente à cesariana, a prevalência da noctúria foi de 40,5\% e da urgência e urge-incontinência de 35,7\%. A análise da associação entre a presença de sintomas urinários irritativos (SUI) após o parto e a via de parto exclusiva não mostrou diferença significativa (Tabela 1 ).

O último parto foi cesárea em 53 mulheres (44,2\%) sendo, deste total, 26,4\% cesarianas eletivas. Entre as 67 (55,8\%) que tiveram partos via vaginal, 19,4\% foi com fórcipe e 73,1\% com episiotomia. A Tabela 2 apresenta os resultados da análise da associação entre a presença de SUI após o parto e a realização da episiotomia, o uso de fórcipe, o peso do RN e a idade materna no último parto. Não foram observadas diferenças significativas.

Em seguida, foi observada maior ocorrência da urgência (66,7\%), urge-incontinência (60\%), e noctúria (73,3\%) em multíparas com quatro ou mais partos quando comparado às primíparas. Entretanto, não houve diferença significativa na associação entre a presença de SUI após o parto e a paridade (Tabela 3). 
Tabela 2 - Distribuição percentual dos sintomas urinários irritativos após o parto segundo a realização da episiotomia, uso de fórcipe, peso do recém-nascido $(R N)$ e idade materna no parto $(n=120)$

\begin{tabular}{|c|c|c|c|c|c|c|c|c|}
\hline \multirow[b]{3}{*}{ Episiotomia } & \multicolumn{8}{|c|}{ Sintomas urinários irritativos } \\
\hline & \multicolumn{2}{|c|}{$\begin{array}{l}\text { Urgência } \\
\text { Sim }\end{array}$} & \multicolumn{2}{|c|}{$\begin{array}{l}\text { Urge-incontinência } \\
\text { Sim }\end{array}$} & \multicolumn{2}{|c|}{$\begin{array}{l}\text { Frequência miccional } \\
\text { Sim }\end{array}$} & \multicolumn{2}{|c|}{$\begin{array}{l}\text { Noctúria } \\
\text { Sim }\end{array}$} \\
\hline & n (\%) & $\begin{array}{l}\text { Valor-p } \\
0,1032\end{array}$ & $\mathrm{n}(\%)$ & $\begin{array}{l}\text { Valor-p } \\
0,0613\end{array}$ & $\mathrm{n}(\%)$ & $\begin{array}{l}\text { Valor-p } \\
0,3272\end{array}$ & $\mathrm{n}(\%)$ & $\begin{array}{l}\text { Valor-p } \\
0,5317\end{array}$ \\
\hline Sim & $19(38,8)$ & & $15(30,6)$ & & $13(26,5$ & & $23(46,9)$ & \\
\hline Não & $11(61,1)$ & & $10(55,6)$ & & $7(38,9)$ & & $10(55,6)$ & \\
\hline Uso de fórcipe & & 0,9774 & & $1,0000 *$ & & $0,4897^{*}$ & & $0,6727 *$ \\
\hline Sim & $5(38,5)$ & & $2(15,4)$ & & $11(84,6$ & & $12(92,3)$ & \\
\hline Não & $21(38,9)$ & & $11(20,4)$ & & $39(72,2$ & & $45(83,3)$ & \\
\hline Peso do RN & & $0,2370^{*}$ & & $0,4261^{*}$ & & $0,0989 *$ & & $0,7039 *$ \\
\hline$<4000 \mathrm{~g}$ & $47(41,6)$ & & $42(37,2)$ & & $30(26,5$ & & $52(46,0)$ & \\
\hline$\geq 4000 \mathrm{~g}$ & $5(71,4)$ & & $4(57,1)$ & & $4(57,1)$ & & $4(57,1)$ & \\
\hline Idade materna & & 0,5333 & & 0,3286 & & 0,6035 & & 0,3479 \\
\hline$<25$ anos & $22(40,7)$ & & $17(31,5)$ & & $13(24,1$ & & $23(42,6)$ & \\
\hline $25-35$ anos & $22(42,3)$ & & $22(42,3)$ & & $16(30,8$ & & $24(46,2)$ & \\
\hline$>35$ anos & $8(57,1)$ & & $7(50,0)$ & & $5(35,7)$ & & $9(64,3)$ & \\
\hline
\end{tabular}

Teste Qui quadrado

*Teste Exato de Fisher

Tabela 3 - Distribuição percentual dos sintomas urinários irritativos (SUI) após o parto segundo a paridade $(n=120)$

\begin{tabular}{|c|c|c|c|c|c|c|c|}
\hline \multirow[t]{2}{*}{ SUI após o parto } & \multicolumn{2}{|c|}{$\begin{array}{l}\text { Primíparas } \\
(\mathrm{n}=45)\end{array}$} & \multicolumn{2}{|c|}{$\begin{array}{l}\text { Multíparas 2-3 } \\
(\mathrm{n}=60)\end{array}$} & \multicolumn{2}{|c|}{$\begin{array}{c}\text { Multípara } \geq 4 \\
(\mathrm{n}=15)\end{array}$} & \multirow[b]{2}{*}{ Valor-p } \\
\hline & $\bar{n}$ & $(\%)$ & $n$ & $(\%)$ & $\mathrm{n}$ & $(\%)$ & \\
\hline Urgência & & & & & & & 0,1381 \\
\hline Sim & 17 & $(37,8)$ & 25 & $(41,7)$ & 10 & $(66,7)$ & \\
\hline Não & 28 & $(62,2)$ & 35 & $(58,3)$ & 5 & $(33,3)$ & \\
\hline Urge-incontinência & & & & & & & 0,1373 \\
\hline $\operatorname{Sim}$ & 14 & $(31,3)$ & 23 & $(38,3)$ & 9 & $(60,0)$ & \\
\hline Não & 31 & $(68,9)$ & 37 & $(61,7)$ & 6 & $(40,0)$ & \\
\hline Frequência miccional & & & & & & & 0,5630 \\
\hline $\operatorname{Sim}$ & 12 & $(26,7)$ & 16 & $(26,7)$ & 6 & $(40,0)$ & \\
\hline Não & 33 & $(73,3)$ & 44 & $(73,3)$ & 9 & $(60,0)$ & \\
\hline Noctúria & & & & & & & $0,0978^{*}$ \\
\hline Sim & 20 & $(44,4)$ & 25 & $(41,7)$ & 11 & $(73,3)$ & \\
\hline Não & 25 & $(55,6)$ & 35 & $(58,3)$ & 4 & $(26,7)$ & \\
\hline Enurese noturna & & & & & & & $0,3768^{*}$ \\
\hline Sim & 2 & $(4,4)$ & 6 & $(10,0)$ & 2 & $(13,3)$ & \\
\hline Não & 43 & $(95,6)$ & 54 & $(90,0)$ & 13 & $(86,7)$ & \\
\hline
\end{tabular}

Teste Qui quadrado

*Teste Exato de Fisher

\section{Discussão}

Mulheres de todas as idades referem a ocorrência de SUI. Em estudo realizado com 17.080 mulheres com mais de 18 anos na França, Alemanha, Inglaterra e Espanha, a prevalência da incontinência urinária foi de 35\%. No grupo entre 18 e 44 anos ( $n=2305)$, a presença da incontinência urinária de esforço (IUE) foi maior (39\%), seguida da urge-incontinência $(22 \%)^{19}$.

O trauma obstétrico parece determinar a ocorrência de sintomas do trato urinário inferior após o parto, alcançando índice de $30 \%$ em primíparas ${ }^{20}$. O desencadeamento da urgência miccional ${ }^{2}$ e da urge-incontinência ${ }^{5}$ após o parto vaginal foi associado ao impacto do parto sobre as estruturas do assoalho pélvico. A redução da força de contração da musculatura estriada do assoalho pélvico predispõe ao acúmulo de determinada quantidade de urina na uretra proximal que estimula a contração involuntária do músculo detrusor ${ }^{21}$. Guarisi et $a^{22}$, por meio da avaliação urodinâmica, observaram hiperatividade do detrusor quatro vezes mais freqüente em mulheres que haviam tido via exclusiva de parto vaginal. 
A cesariana eletiva aparece na literatura como um procedimento protetor para as estruturas do assoalho pélvico, prevenindo o desencadeamento de sintomas urinários ${ }^{11}$. Entretanto, este efeito protetor parece limitar-se aos primeiros meses do período pós-parto. Dois anos depois do parto, não foi encontrada diferença significativa entre a via de parto e sintomas urinários, alterações estruturais no segmento pélvico-lombar, disfunção sexual e planejamento de outra gestação ${ }^{12}$. Primíparas, que tiveram parto via vaginal, apresentaram maior ocorrência da urgência e da urge-incontinência no puerpério, comparado às mulheres submetidas à cesariana. Entretanto, um ano depois do parto, esta diferença não se manteve significativa ${ }^{8}$. Assim como Chaliha et al. ${ }^{20}$, neste estudo, não foi observada associação significativa entre a presença dos sintomas urinários irritativos após o parto e a via exclusiva de parto (vaginal ou cesárea).

Após os anos reprodutivos da mulher, a via exclusiva de parto cesárea não parece também prevenir o desencadeamento da incontinência urinária e a gestação per se pode apresentar risco superior para a ocorrência do sintoma ao longo da vida ${ }^{16}$.

Chaliha et al. $^{7}$ encontraram porcentagens de frequência miccional $(81,1 \%)$, noctúria $(67,6 \%)$ e urgência $(22,9 \%)$ superiores na gestação quando comparadas ao período pósparto, $22,4 \%, 4,4 \%$ e 7,8\%, respectivamente. Ao comparar o impacto da gestação e do parto no desencadeamento de SUI, Scarpa et al. ${ }^{23}$ observaram que a gestação, mais do que o parto, foi responsável pelo desencadeamento da IUE e noctúria. Entre as mulheres com frequência miccional na gestação, 67,4\% tornaram-se assintomáticas após o parto e com relação às mulheres com urgência e urge-incontinência durante a gestação, $62,7 \%$ e $68 \%$, respectivamente, persistiram sintomáticas três anos após o parto ${ }^{23}$. No terceiro trimestre gestacional, a alta prevalência de SUI tem sido relacionada às alterações mecânicas, como o efeito da pressão do útero gravídico sobre a bexiga, associado ao aumento da sua sensibilidade e à diminuição significativa da capacidade vesical ${ }^{24,25}$. A presença de SUI na gestação e após o parto esteve associada a modificações de parâmetros urodinâmicos, como a capacidade cistométrica máxima e o primeiro desejo miccional, que se encontram abaixo dos limites definidos para mulheres não grávidas ${ }^{25}$.

Assim como outros autores ${ }^{2,6}$, não notamos associação entre SUI após o parto e a paridade. A avaliação dos sintomas irritativos neste estudo foi realizada de forma subjetiva. Foram incluídas primíparas e multíparas, porém a amostra inferior nos subgrupos pode ter limitado o resultado da análise.

O uso de fórcipe durante o parto vaginal foi associado ao aumento da frequência de urge-incontinência após o parto ${ }^{4}$. Com relação à idade materna, Altman et al. ${ }^{2}$ concluíram que esta variável não determinou a ocorrência da urgência miccional após o parto. Neste estudo, não houve associação significativa entre qualquer sintoma irritativo após o parto e a realização da episiotomia, o uso de fórcipe e a idade materna. Nos Estados Unidos, entre 1999 e 2000, houve uma redução de 56\% da realização de episiotomia ${ }^{26}$.

Rortveit et al. ${ }^{11}$ notaram que as mulheres que tiveram pelo menos um filho com mais de 4000 g relatavam com maior frequência distúrbios urinários. Neste estudo, a análise da associação entre o peso do RN ( $\geq 4000 g$ ) e a presença de SUI após o parto não mostrou diferença significativa. O pequeno número de casos de recém-nascidos com peso superior à 4000g, provavelmente decorrente do baixo nível socioeconômico das mulheres que compõem nossa amostra, pode representar um fator de erro na análise desta variável.

Muitas mulheres ainda relutam em relatar a presença de SUI aos seus clínicos ${ }^{19}$, consequentemente poucas ainda se beneficiam da terapêutica conservadora. Na literatura, autores demonstraram que a realização de exercícios da musculatura do assoalho pélvico após o parto, sob supervisão fisioterápica, pode fortalecer e restaurar a força da contração voluntária do músculo elevador do ânus, promovendo a melhora dos sintomas urinários ${ }^{27,28}$.

Na prática obstétrica contemporânea, múltiplos fatores contribuem na decisão de obstetras e gestantes quanto à via de parto. Os resultados deste estudo sugerem que o impacto do parto vaginal e a paridade não foram fatores de risco determinantes para a disfunção do trato urinário inferior após o parto representada pelos sintomas urinários irritativos. Esta informação poderá contribuir nas decisões da gestante concernentes a seu parto.

\section{Conclusão}

Não houve associação entre a presença de sintomas urinários irritativos três anos após o parto e a via de parto exclusivamente vaginal ou cesárea, sugerindo que a gestação, independente da via de parto, pode promover o desencadeamento de SUI. Também não encontramos associação entre os SUI e a paridade, a idade materna no parto, peso do RN, realização da episiotomia e uso de fórcipe.

\section{Conflito de interesse: não há}

\section{SUMMARY}

IRRITATIVE BLADDER SYMPTOMS AFTER VAGINAL DELIVERY OR C-SECTION

OBJECTIVE. This study intended to investigate the frequency of irritative bladder symptoms three years after delivery in women previously interviewed in the third trimester of pregnancy and its correlation to mode of delivery, parity, maternal age, birth weight, episiotomy and forceps.

METHODS. From 340 women previously evaluated at the third trimester of pregnancy, 120 were interviewed three years after delivery, between June and October 2006. Correlation of postpartum irritative bladder symptoms and mode of delivery (exclusively vaginal or c-section), parity, maternal age, birth weight, episiotomy and forceps was analyzed. Associations between irritative bladder symptoms and obstetric parameters were assessed by the Fisher's exact test and Chi-square $(p<0.05)$.

RESULTS. The study group consisted of $37.5 \%$ primiparous and $62.5 \%$ multiparous women. Mode of delivery was exclusively vaginal in 53 women and exclusively c-section in 42. No statistical difference was found between irritative bladder symptoms and mode of delivery and parity. No statistical difference was found between irritative symptoms and maternal age ( $>35$ ), birth weight ( $\geq 4000 \mathrm{~g}$ ), episiotomy and forceps.

CONCLUSION. After childbirth, dysfunction of the lower urinary tract, characterized by irritative bladder symptoms, was not associated with mode of delivery, parity, maternal age, birth 
weight, episiotomy and forceps. [Rev Assoc Med Bras 2009; 55(4): 416-20]

KEY WORDS: Irritative Bladder Symptoms. Parity. Delivery. Cesarean Section. Episiotomy.

\section{REFERÊNCIAS}

1. Fritel X, Fauconnier A, Levet C, Bénifla JL. Stress urinary incontinence 4 years after the first delivery: a retrospective cohort survey. Acta Obstet Gynecol Scand. 2004;83:941-5

2. Altman D, Ekström A, Gustafsson C, López A, Falconer C, Zellerström J. Risk of urinary incontinence after childbirth: a 10-years prospective cohort study. Obstet Gynecol. 2006;108:873-8

3. Oliveira E, Takano CC, Sartori JP, Araújo MP, Pimentel SHC, Sartori MGF, et al. Trato urinário, assoalho pélvico e ciclo gravídico-puerperal. Femina.2007;35:89-94.

4. Casey BM, Schaffer JI, Bloom SL, Heartwell SF, Mcintire DD, Leveno KJ Obstetric antecedents for postpartum pelvic floor dysfunction. Am J Obstet Gynecol. 2005;192:1655-62.

5. Fenner DE, Genberg B, Brahma P, Marek L, Delancey JOL. Fecal and urinary incontinence after vaginal delivery with anal sphincter disruption in an obstetrics unit in the United States. Am J Obstet Gynecol. 2003; 189:1543-50.

6. Rogers RG, Leeman LM, Migliaccio L, Albers LL. Does the severity of spontaneous genital tract trauma affect postpartum pelvic floor function? Int Urogynecol J. 2008; 19:429-35.

7. Chaliha C, Kalia V, Stanton SL, Monga A, Sultan AH. Antenatal prediction of postpartum urinary and fecal incontinence. Obstet Gynecol. 1999;94:689-94.

8. Van Brummen HJ, Bruinse HW, Van de Pol G, Heintz APM, Van der Vaart CH. The effect of vaginal and cesarean delivery on lower urinary tract symptoms: what makes the difference? Int Urogynecol J. 2007;18:133-9.

9. Schytt E, Lindmark G, Waldenström U. Symptoms of stress incontinence 1 year after childbirth: prevalence and predictors in a national Swedish sample. Acta Obstet Gynecol. Scand.2004;83:928-36.

10. Scarpa KP, Herrmann V, Palma PC, Riccetto CL, Morais SS. Prevalência de sintomas urinários no terceiro trimestre da gestação. Rev Assoc Med Bras. 2006;52:153-6.

11. Rortveit G, Daltveit AK, Hannestad YS, Hunskaar S. Vaginal delivery parameters and urinary incontinence: the Norwegian EPINCONT study. Am J Obstet Gynecol. 2003;189:1268-74

12. Hanna ME, Whyte H, Hanna WJ, Hewson S, Amankwah K, Cheng M, et al Maternal outcomes at 2 years after planned cesarean section versus planned vaginal birth for breech presentation at term: the international randomized Term Breech Trial. Am J Obstet Gynecol. 2004;191:917-27.
13. Barros FC, Vaughan JP, Victoria CG, Huttly SRA. Epidemic of cesarean sections in Brazil. Lancet. 1991;338:167.

14. Brasil. Ministério da Saúde. J Febrasgo. 2004;11:4-6.

15. Potter JE, Hopkins K, Faúndes A, Perpétuo I. Women's autonomy and scheduled cesarean sections in Brazil: a cautionary tale. Birth. 2008;35:33-40.

16. Faúndes A, Guarisi T, Pinto-Neto AM. The risk of urinary incontinence of parous women who delivered only by cesarean section. Int J Gynecol Obstet. $2001 ; 72: 41-6$.

17. Abrams P, Cardozo L, Fall M, Griffiths D, Rosier P, Ulmsten U, et al. The standartization of terminology of lower urinary tract function: report from the standartization sub committee of the international continence society. Neurourol Urodyn.2002; 21:1676-8.

18. Snedecor WG, Cochram WG. Statistical methods. $8^{\text {th }}$ ed. lowa State; 1989.

19. Hunskaar S, Lose G, Sykes D, Voss S. The prevalence of urinary incontinence in women in four European countries. BJU Int. 2004;93:324-30.

20. Chaliha C, Khullar V, Stanton SL, Monga A, Sultan AH. Urinary symptoms in pregnancy: are they useful for diagnosis? $\mathrm{Br} J$ Obstet Gynecol. 2002;109:1181-3

21. Rosenzweig BA, Pushkin S, Blumenfeld D, Bhatia NN. Prevalence of abnormal urodynamic test results in continent women with severe genitourinary prolapse. Obstet Gynecol. 1992;79:539-42.

22. Guarisi T, Pinto-Neto AM, Herrmann V, Faúndes A. Urodynamics in climateric women with urinary incontinence: correlation with route of delivery. Int Urogynecol J.2002; 13:366-71.

23. Scarpa KP, Herrmann V, Palma PC, Riccetto CL, Morais SS. Sintomas do trato urinário inferior três anos após o parto: estudo prospectivo. Rev Bras.Ginecol. Obstet. 2008;30:355-9.

24. Van Brummen HJ, Bruinse HW, Van De Pol G, Heintz APM, Van Der Vaart $\mathrm{CH}$. What is the effect of overactive bladder symptoms on woman's quality of life during and after first pregnancy? BJU Int. 2006;97:296-300.

25. Chaliha K, Bland JM, Monga A, Stanton SL, Sultan AH. Pregnancy and delivery: a urodynamic viewpoint. Br J Obstet Gynaecol. 2000;107:1354-9.

26. Clemons JL, Towers GD, McClure GB, O'Boyle AL. Decreased anal sphincter lacerations associated with restrictive episiotomy use. Am J Obstet Gynecol. 2005; 192:1620-3.

27. Wein AJ. Diagnosis and treatment of overactive bladder. Urology. 2003; $62(5 B): 20-7$

28. Van Brummen $\mathrm{HJ}$, Bruinse HW, Van De Pol G, Heintz APM Van Der Vaart $\mathrm{CH}$. Bothersome lower urinary tract symptoms 1 year after first delivery: prevalence and effect of childbirth. BJU Int. 2006;98:89-95.

Artigo recebido: 16/04/08 Aceito para publicação: 16/02/09 